\title{
Energetic proton depletion near Io: atmospheric charge exchange and inhomogeneous magnetic and electric fields
}

\author{
Christiaan van Buchem ${ }^{1,2}$, Hans Huybrighs ${ }^{1}$, Aljona Blöcker ${ }^{3}$, Vincent Dols ${ }^{4}$, Olivier Witasse ${ }^{1}$, and \\ Yamila Miguel $^{2}$ \\ ${ }^{1}$ ESA, ESTEC, Noordwijk, Netherlands (christiaanvanbuchem@gmail.com) \\ ${ }^{2}$ Leiden University, Leiden, the Netherlands \\ ${ }^{3} \mathrm{KTH}$, Royal Institute of Technology, Stockholm. Sweden \\ ${ }^{4}$ LASP, Laboratory of Atmospheric and Space Physics, Colorado, USA
}

The flux of energetic protons ( $80 \mathrm{keV}-1.04 \mathrm{Mev}$ ) near the Galilean moons was measured by the Energetic Particle Detector (EPD) on the Galileo mission (1995 - 2003). Near Galilean moon, such as Io and Europa, depletions of the energetic proton flux, of several orders of magnitude, were observed.

Such energetic proton depletions can be caused by the precipitation of these particles onto the moon's surface or charge exchange with the neutral atmosphere. In order to interpret the depletion features in the EPD data, a Monte Carlo particle tracing code has been developed by $(H$. L. F. Huybrighs et al., 2020; Hans L. F. Huybrighs et al., 2017; Hans Leo Frans Huybrighs, 2018). The expected flux of the energetic ions is simulated under different scenarios, for three Galileo flybys of Io (I24, I27, and I31), including with and without an atmosphere and inhomogeneous magnetic and electric fields. By comparing the simulated distribution to the EPD data, the cause of the depletion features can be investigated.

The following causes of energetic proton depletion near Io are identified:

- Some depletions are consistent with atmospheric charge exchange for flybys I24, I27, and I31.

- Some of depletions coincide with the inhomogeneous fields produced in the MHD model by (Dols et al., 2012) for flybys I24, I27, and I31.

- For I24 the depletions are consistent with a two component atmosphere: a dense low scale height atmosphere and an extended corona described by a low surface density but a large scale height as presented by (Blöcker et al., 2018).

Furthermore, latitudinal and longitudinal dependencies in the atmospheric models for Io are investigated for all three aforementioned flybys.

\section{Bibliography}

- Blöcker, A., Saur, J., Roth, L., \& Strobel, D. F. (2018). MHD Modeling of the Plasma Interaction With Io's Asymmetric Atmosphere. Journal of Geophysical Research: Space Physics, 123(11), 9286-9311. https://doi.org/10.1029/2018JA025747 
- Dols, V., Delamere, P. A., Bagenal, F., Kurth, W. S., \& Paterson, W. R. (2012). Asymmetry of Io's outer atmosphere: Constraints from five Galileo flybys. Journal of Geophysical Research: Planets, 117(E10). https://doi.org/10.1029/2012JE004076

- Huybrighs, H. L. F., Roussos, E., Blöcker, A., Krupp, N., Futaana, Y., Barabash, S., et al. (2020). An Active Plume Eruption on Europa During Galileo Flyby E26 as Indicated by Energetic Proton Depletions. Geophysical Research Letters, 47(10). https://doi.org/10.1029/2020gl087806

- Huybrighs, Hans L. F., Futaana, Y., Barabash, S., Wieser, M., Wurz, P., Krupp, N., et al. (2017). On the in-situ detectability of Europa's water vapour plumes from a flyby mission. https://doi.org/10.1016/j.icarus.2016.10.026

- Huybrighs, Hans Leo Frans. (2018). A search for signatures of Europa's atmosphere and plumes in Galileo charged particle data. Retrieved from http://arxiv.org/abs/1812.11215 